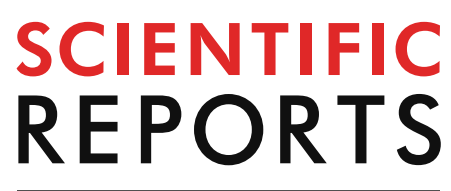

natureresearch

Check for updates

\title{
Cytochrome P450 inhibition potential and initial genotoxic evaluation of 14-0-[(4,6-diaminop yrimidine-2-yl)thioacetyl] mutilin
}

\author{
Yunxing Fu ${ }^{1,2}$, Yunpeng $\mathrm{Yi}^{1}$, Yuan Fan ${ }^{1}$ \& Ruofeng Shang ${ }^{1 \bowtie}$
}

14-0-[(4,6-Diaminopyrimidine-2-yl)thioacetyl] mutilin (DPTM) is a promising drug candidate with excellent antibacterial activity against Gram-positive bacteria. The present study was designed to characterize its Cytochrome P450 (CYP) enzymes inhibition activities and the genotoxicity with the standard Ames test. We determined the inhibitory effects of DPTM on CYP1A2, CYP2D1/6, CYP2E1, CYP2C11/9 and CYP3A/4 in rat liver microsomes (RLMs) and in human liver microsomes (HLMs). The mRNA expressions of the above CYP isoforms and their transcriptional regulators were also evaluated using the Hep $\mathrm{G} 2$ cell model. The results showed DPTM exhibited a moderate inhibitory potential against CYP3A/4 ( $\left(\mathrm{C}_{50}\right.$ values of $10 \pm 2$ and $8 \pm 2 \mu \mathrm{M}$, respectively) and weak against the other CYP enzymes based on their $\mathrm{IC}_{50}$ values. Compared to the control, CYP isoforms and their transcriptional regulators mRNA expressions significantly increased when the Hep G2 cells were treated with DPTM for a certain period of time. In the Ames test, Salmonella strains TA97, TA98, TA100, TA102 and TA1535 were treated with or without the metabolic activation (S9). Analysis showed the average number of revertant colonies per plate was less in double in the groups treated with DPTM than that in the negative control plate and showed no dose-related increase.

Pleuromutilin (Fig. 1), a 5-6-8 tricycle diterpene, was first discovered and isolated from two basidiomycete species in $1951^{1}$. Pleuromutilin class of antibiotics selectively inhibits bacterial protein synthesis by interacting with the peptidyl transferase centre (PTC) of prokaryotic ribosomes at the A- and P-site through hydrophobic interactions and hydrogen bond $\mathrm{s}^{2-4}$. The modifications of pleuromutilin side chain led to tiamulin (Fig. 1) and valnemulin (Fig. 1) for veterinary use ${ }^{5,6}$. Retapamulin (Fig. 1) is a topically administered pleuromutilin that was first approved for human use in $2007^{7,8}$. Lefamulin (BC-3781, Fig. 1) developed by Nabriva Therapeutics was approved by FDA for human use in $2019^{9,10}$.

Azamulin (Fig. 1), an azole pleuromutilin derivative designed for human use, has entered phase I clinical trials in volunteers in $1980 \mathrm{~s}^{11}$. Unfortunately, azamulin did not undergo further clinical trials because of its Cytochrome P450 (CYP) inhibition and limited bioavailability ${ }^{12,13}$. Tiamulin is also a potent inhibitor and inducer of CYP, via the formation of a metabolic intermediate complex ${ }^{14}$. Lefamulin showed relative high inhibition for CYP3A4 with $\mathrm{IC}_{50}<5 \mu \mathrm{M}^{15}$. Therefore, the investigation of inhibition potency of pleuromutilin analogues against CYP was subsequently emphasized to avoid the risk for development a new drug.

A novel pleuromutilin derivative, 14-O-[(4,6-Diaminopyrimidine-2-yl)thioacetyl] mutilin (DPTM, Fig. 1), was synthesized and reported in ours previous work ${ }^{16}$. This compound showed the excellent in vitro activities against Gram-positive bacteria, including methicillin-resistant Staphylococcus aureus (MRSA), Staphylococcus aureus (S. aureus), Bacillus subtilis (B. subtilis), as well as in vivo activity using systemic infection mode in mice $^{16}$. As previous studies reported that pleuromutilin analogues showed high CYP inhibition effect ${ }^{17,18}$, we evaluated the inhibitory effects of DPTM on the major CYP enzymes, including CYP1A2, CYP2D1, CYP2E1, CYP2C11 and CYP3A in rat liver microsomes (RLMs) and CYP1A2, CYP2D6, CYP2E1, CYP2C9 and CYP3A4 in human liver microsomes (HLMs). Furthermore, mRNA expressions of CYP enzymes and their transcriptional

${ }^{1}$ Key Laboratory of New Animal Drug Project, Gansu Province, Key Laboratory of Veterinary Pharmaceutical Development, Ministry of Agriculture and Rural Affairs, Lanzhou Institute of Husbandry and Pharmaceutical Sciences of CAAS, Qilihe District, No. 335, Lanzhou 730050, People's Republic of China. ${ }^{2}$ Henan University of Animal Husbandry and Economy, Zhengzhou 450046, People's Republic of China. ${ }^{\square}$ email: shangrf1974@163.com 


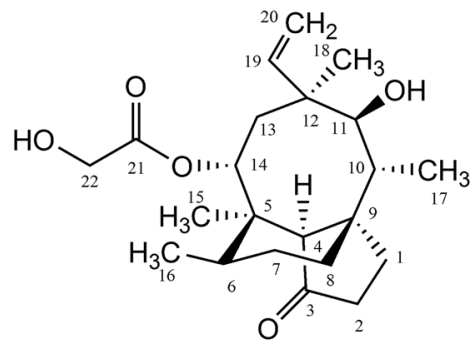

Pleuromutilin 1

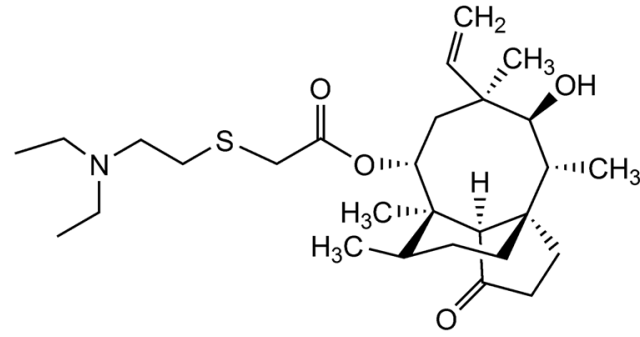

Tiamulin

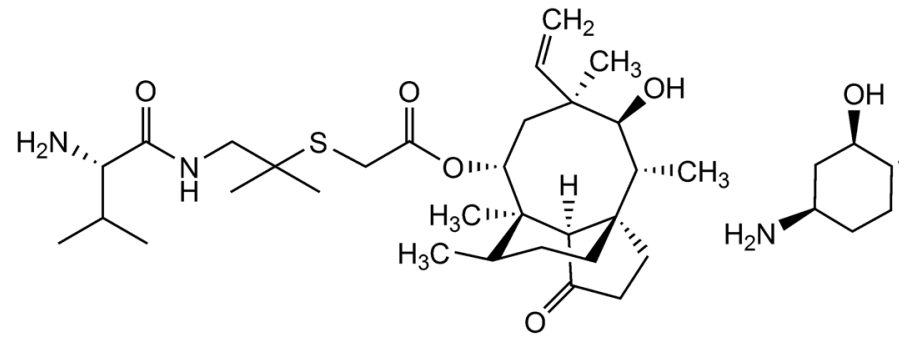

Valunemulin

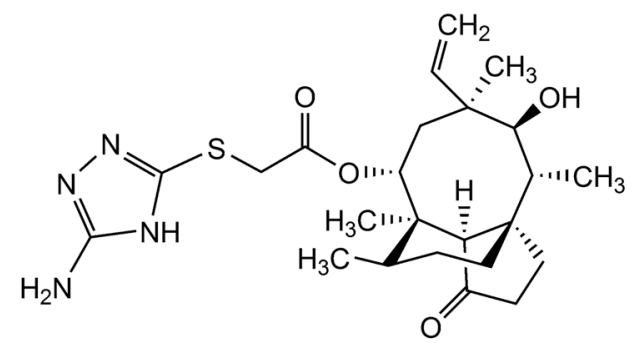

Azamulin

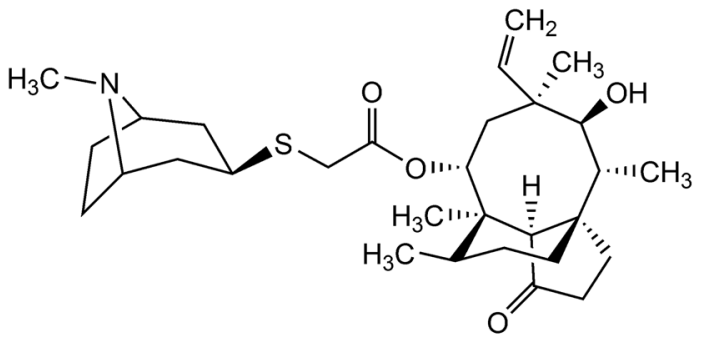

Retapamulin

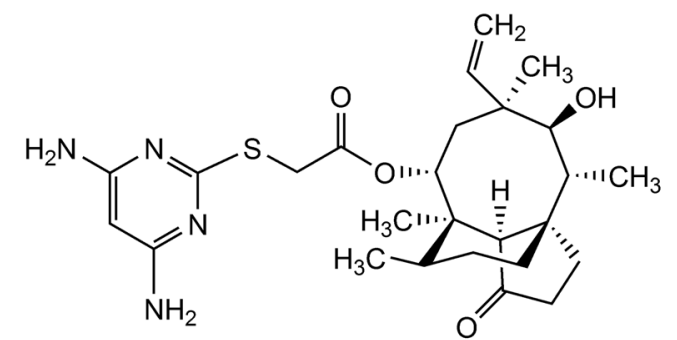

DPTM

Figure 1. Structures of pleuromutilin, pleuromutilin drugs and 14-O-[(4,6-Diaminopyrimidine-2-yl) thioacetyl] mutilin (DPTM).

regulators, including the aryl hydrocarbon receptor (AhR), the pregnane X receptor (PXR) and the peroxisome proliferator-activated receptor (PPAR) were investigated after treatment by DPTM using the human HepG2 cell-line. Moreover, the genotoxic properties of DPTM were also assessed by the standard Ames test with and without metabolic activation (S9).

\section{Results}

Cytotoxicity. Using the MTT (3-(4,5-dimethyl-2-thiazolyl)-2,5-diphenyl-2H-tetrazolium bromide) assay, the cytotoxic potential of DPTM was evaluated on Hep G2 cells. The viability percentages of cells treated with different dilutions of DPTM were shown in Fig. 2. The result was represented as the $50 \%$ inhibition $\left(\mathrm{IC}_{50}\right.$ ) value. On the whole, along with the increase of concentrations of DPTM, the cell viability became lower with the $\mathrm{IC}_{50}$ value of $10 \pm 2 \mathrm{mM}$.

Effect of drug on cytochrome P450. We used cocktail method to assess the direct inhibitory potential of DPTM on CYPs in RLMs and HLMs. The determined $\mathrm{IC}_{50}$ values with $95 \%$ confidence intervals from eight- 


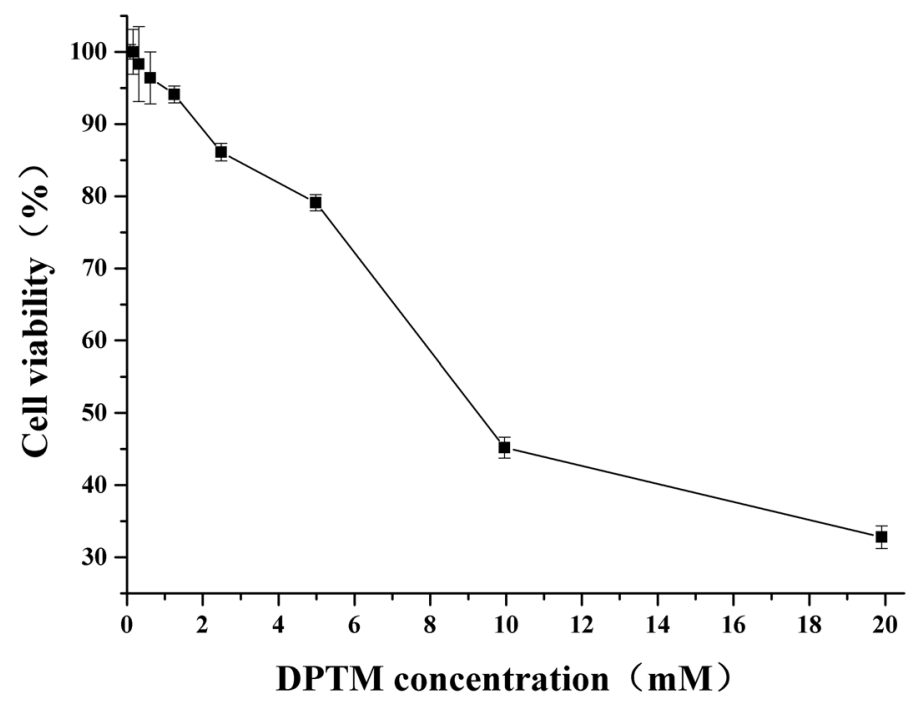

Figure 2. Cytotoxicity of DPTM for Hep G2 cells tested by MTT methodology. Each data point represents the mean value $\pm \mathrm{SD}(\mathrm{n}=3)$.

A

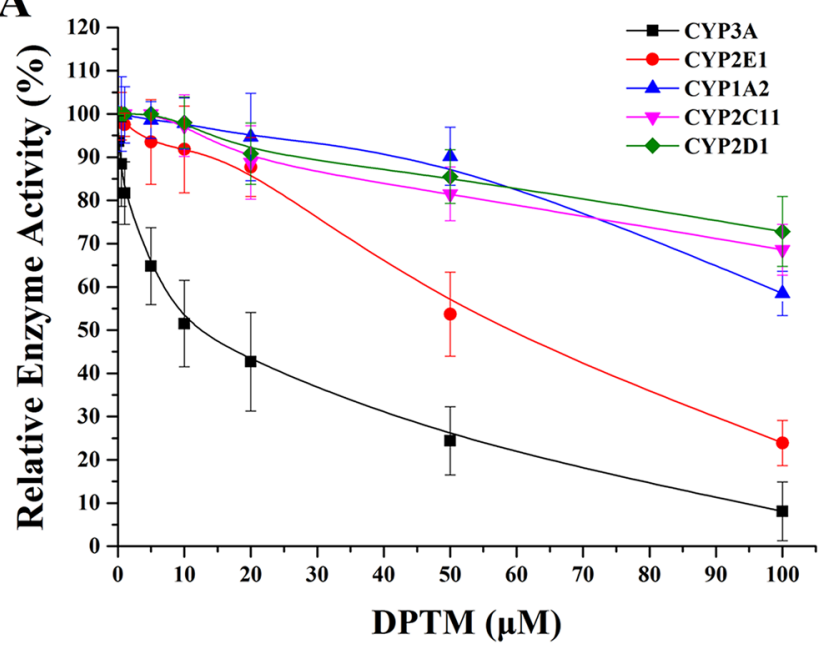

B

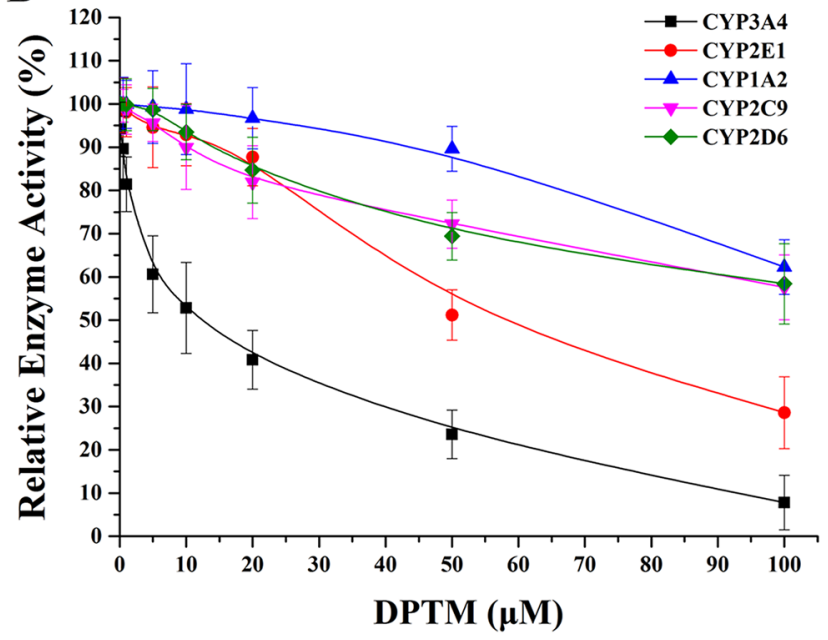

Figure 3. Inhibitory effects of DPTM on CYP isoforms in RLMs (A) and in HLMs (B). Each data point represents the mean value $\pm \mathrm{SD}(\mathrm{n}=3)$.

\begin{tabular}{|l|l|l|l|}
\hline Isoform in RLMs & $\mathbf{I C}_{\mathbf{5 0}}$ values $(\boldsymbol{\mu M})$ & Isoform in HLMs & $\mathbf{I C}_{\mathbf{5 0}}$ values $(\boldsymbol{\mu M})$ \\
\hline CYP1A2 & $>100$ & CYP1A2 & $>100$ \\
\hline CYP2D1 & $>100$ & CYP2D6 & $>100$ \\
\hline CYP2E1 & $61 \pm 8$ & CYP2E1 & $67 \pm 6$ \\
\hline CYP2C11 & $>100$ & CYP2C9 & $>100$ \\
\hline CYP3A & $10 \pm 2$ & CYP3A4 & $8 \pm 2$ \\
\hline
\end{tabular}

Table 1. Inhibitory effects of DPTM on CYP isoforms in RLMs and HLMs.

point concentration-response curves (Fig. 3) were listed in the Table 1. Ketoconazole and fluvoxamine used as the positive controls for the inhibition of CYP3A4 and CYP1A2, respectively, showed strong activities with $\mathrm{IC}_{50}$ values $\left(0.18\right.$ and $0.33 \mu \mathrm{M}$, respectively) within the reference ranges ${ }^{19}$. In RLMs and HLMs, DPTM exhibited moderate inhibitory potentials against CYP3A and CYP $3 \mathrm{~A} 4$ with $\mathrm{IC}_{50}$ values of $10 \pm 2$ and $8 \pm 2 \mu \mathrm{M}$, respectively. In the cases of CYP1A2, CYP2D1/6, CYP2E1 and CYP2C11/9, DPTM showed weak inhibitory effects with $\mathrm{IC}_{50} \geq 61 \pm 8 \mu \mathrm{M}$. 
Effect of DPTM activation on cytochrome P450 mRNA expression. To further investigate the inhibitions of DPTM against CYP isoforms, the expressions of the regulated genes of CYP1A2, CYP2D6, CYP2E1, CYP2C9 and CYP3A4 and their transcriptional regulators (AhR, PXR and PPAR) were evaluated using the Hep G2 cell model. Compared to the control, treatment for 2 and $4 \mathrm{~h}$ with 4.98, 0.16 and $0.08 \mathrm{mM}$ DPTM resulted in significantly increase in CYP1A2, CYP2C9 and CYP3A4 mRNA expressions (Fig. 4A-C) and showed dosedependent effect. When DPTM treated for $12 \mathrm{~h}$ with 4.98 and $0.16 \mathrm{mM}$, CYP2C9 and CYP3A4 mRNA expressions were significantly higher than that of control (Fig. 4B,C), but CYP1A2, CYP2D6 and CYP2E1 mRNA expressions were significantly lowered than that of control when treated for $12 \mathrm{~h}$ (Fig. 4A,D,E). However, no significantly difference of CYP2E1 mRNA level between three doses of DPTM and control was found after treatment for $4 \mathrm{~h}$ (Fig. $4 \mathrm{E}$ ).

Compared to the control, treatment with 4.98, 0.16 and $0.08 \mathrm{mM}$ DPTM for 2 and $4 \mathrm{~h}$ significantly increased the AhR mRNA content, while there was no effect when treated for $12 \mathrm{~h}$ (Fig. 5A). Three doses of DPTM significantly increased the PXR mRNA expression when treated for 4 and $12 \mathrm{~h}$ (Fig. 5B). Compared to the control, three doses of DPTM could significantly increase PPARa mRNA expression when treated for 2,4 and $12 \mathrm{~h}$ (Fig. 5C).

Ames test. The genotoxicity of DPTM was conducted by bacterial reverse mutation assay against five mutant S. typhimurium strains with or without $\mathrm{S} 9$ metabolic activation. The results showed revertant colonies were observed in all positive groups and were much more in double than that induced by five concentrations of DPTM and DMSO (Table 2). However, there was no significantly different between the number of revertant colonies induced by DPTM and the negative control (DMSO), which indicated that DPTM should not be considered as a mutagen ${ }^{20}$. The number of colonies induced by DPTM also showed no dose-dependent effect based on this test.

\section{Discussion}

Drug-drug interaction potential and genotoxic activity of novel drugs are important properties for their development and essential to guide appropriate use in patients. This study evaluated the in vitro CYP enzymes inhibition activities and the genotoxicity of DPTM, a novel pleuromutilin derivative with a substituted pyrimidine moiety.

In the present studies, the purity of DPTM (98.72\%) was below 99.00\%, which was not enough pure to examine its inhibitory effects and genotoxic property. According to the synthetic scheme16 and HPLC chromatography of DPTM (see Supplementary Fig. S1), sodium $p$-toluenesulfonate and $p$-toluenesulfonic acid, the two know genotoxic impurities, showed strongly water soluble and could be completely removed when the reaction mixture was washed with water after the chemical reactions. Pleuromutilin, 4,6-diamino-2-mercaptopyrimidine and 14-O-(p-Toluene-sulfonyloxyacetyl)-mutilin, the main process impurities, could be neglected toxicity properties because they were usually used as pharmaceutical raw materials. Therefore, in spite of the low purity of DPTM, the biased results of its CYP enzymes inhibition activity and the genotoxicity could be negligible.

Before the bioactivities studies on the cellular level, it is necessary to evaluate the cytotoxic potential of a drug. The cell viability against DPTM was $79 \%$ with the concentration of $4.98 \mathrm{mM}$, indicating that DPTM showed lower cell toxicity and could be used for the further studies on the Hep G2 cell model with concentration not more than $4.98 \mathrm{mM}$.

CYP enzymes are very important for the metabolism of a wide range of endogenous compounds, as well as xenobiotics including drugs, environmental pollutants and dietary products ${ }^{21-23}$. Some chemicals affect the disposition of conventional pharmaceuticals through the inhibition of CYP enzymes ${ }^{24,25}$. Therefore, we used the cocktail method to determine the substrate content of CYP1A2, CYP2D1/6, CYP2E1, CYP2C9/11 and CYP3A/4 in RLMs and HLMs for evaluating the inhibition activity of DPTM at different concentrations. Our study showed that DPTM displayed a moderate inhibitory effect on CYP3A4, similar to retapamulin ${ }^{8,26}$, but apparently lower than that of azamulin with $\mathrm{IC}_{50}$ value of $0.24-0.03 \mu \mathrm{M}$, and 14-O-((5-Amino-benzimidazole-2-yl) thioacetyl) mutilin (with $\mathrm{IC}_{50}$ value of $\left.1.69 \mu \mathrm{M}\right)^{12,27}$. However, the selective inhibition for CYP3A4 may be a primary function of the pleuromutilin portion of the molecule ${ }^{12,28}$. Furthermore, the side chain structure of pleuromutilin derivative has some influence on CYP3A4 inhibition profiles ${ }^{29}$.

For further evaluating the inhibition activities of DPTM on the CYP isoforms, we investigated CYP enzymes and their main transcription factors (AhR, PXR and PPAR) mRNA expressions using human HepG2 cell-line. The main transcription factors AhR, PXR and PPAR are responsible for the induction of one to four CYP fami-


DPTM could increase the mRNA expressions of CYP isoforms. However, the significantly decrease of the mRNA expressions of CYP1A2, CYP 2D6 and CYP 2E1 when treated with DPTM for $12 \mathrm{~h}$ (Fig. 4) may be related to the rapid metabolic property of DPTM with half-life $\left(t_{1 / 2}\right)$ of $1.7-1.9 \mathrm{~h}$ in rats after intravenous administration ${ }^{32}$. We speculated that HepG2 cells metabolize DPTM rapidly within a short time. After treatment for $12 \mathrm{~h}$, the majority of prototype drug was changed to one or more new compounds that probably inhibited the metabolic function of the cells. However, this mechanism was obtained on the basis of speculation and should be further confirmed.

The mRNA expressions of transcriptional regulators were also increased when HepG2 cells were treated by DPTM (Fig. 5). Interestingly, this compound displayed the similar effect on the CYP enzymes mRNA expressions. Ames test is a rapid, effective and short-term bacterial reverse mutation assay which is commonly employed as an initial screening for genotoxic activity of chemical substances in $v_{i t r o} 33,34$. The present study was carried out using five S. typhimurium strains TA97, TA98, TA100, TA102 and TA1535 with and without S9 metabolic activation. We did not observe any treatment-related mutagenic activity in the histidine auxotrophy of the $S$. typhimurium strains up to $3.98 \mu \mathrm{mol} / \mathrm{plate}$, indicating that under this study conditions, they do not produce reverse mutations in five $S$. typhimurium strains either in the presence or absence of metabolic activation system. 
A

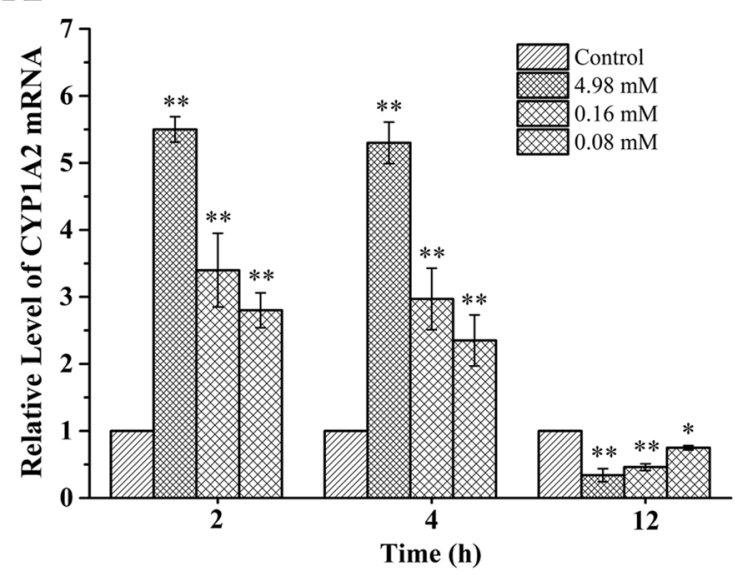

C

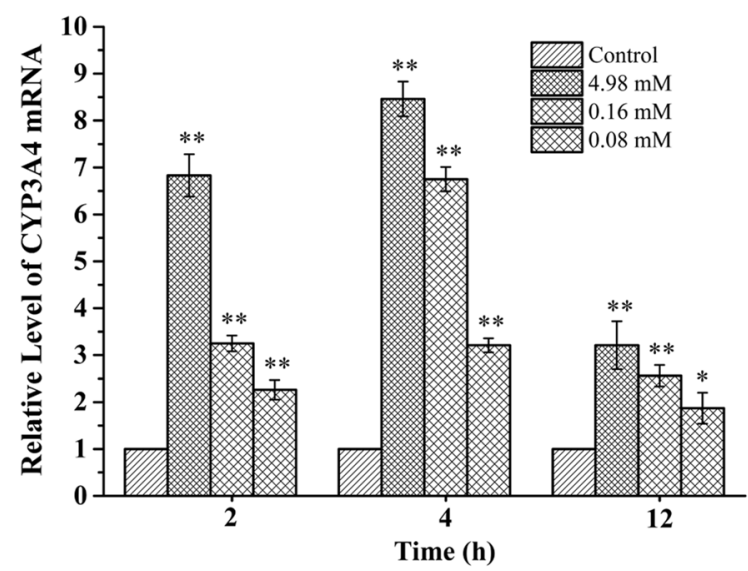

B

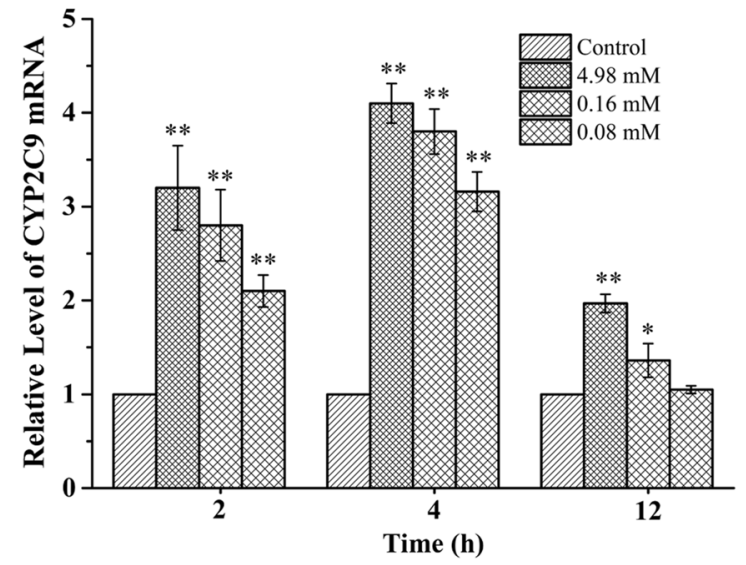

D

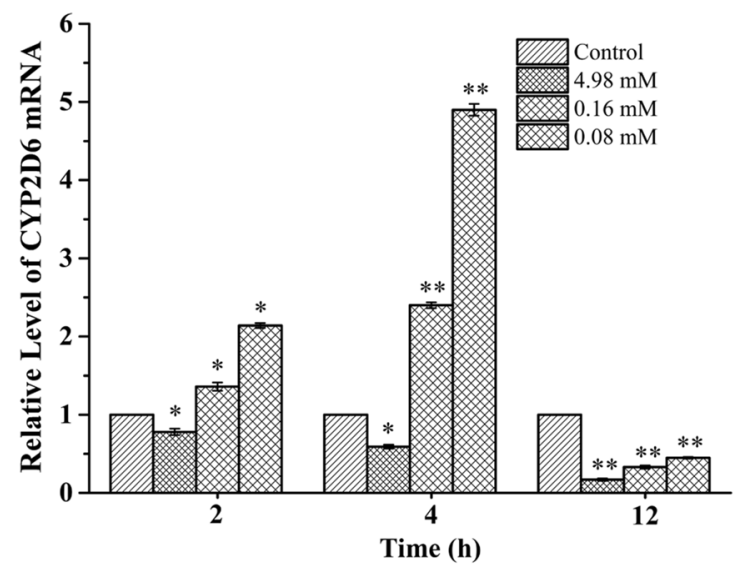

$\mathbf{E}$

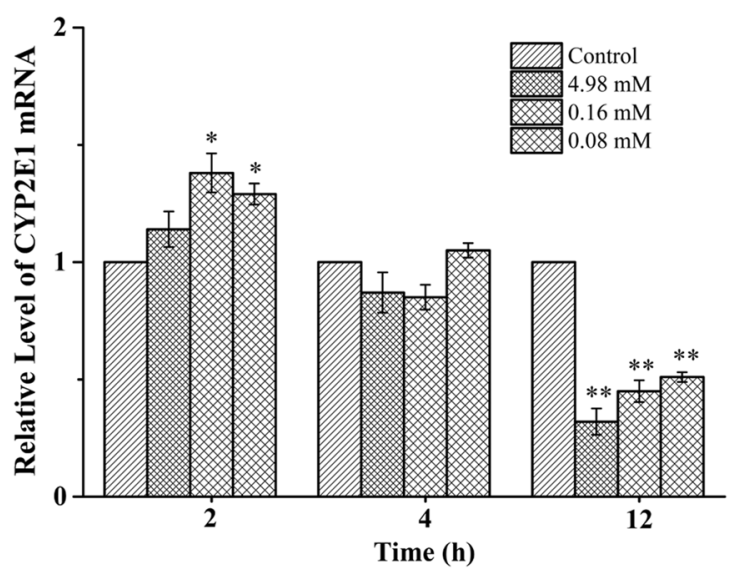

Figure 4. In vitro effect of DPTM on the mRNA expression in Hep G2 cells. CYP 450 enzymes include CYP1A2 (A), CYP2C9 (B), CYP3A4 (C), CYP2D6 (D) and CYP2E1 (E). Data are the mean \pm SD $(n=3)$. ${ }^{\star} P<0.05$ and ${ }^{\star *} P<0.01$ versus the control group.

Furthermore, no linear relationship of number of colonies induced by five doses of DPTM was observed with or without S9.

In conclusion, DPTM displayed moderate inhibitory potentials on CYP3A/4 and weak against CYP1A2, CYP2D1/6, CYP2E1 and CYP2C11/9 in RLMs and HLMs using cocktail method. Furthermore, this compound 
A

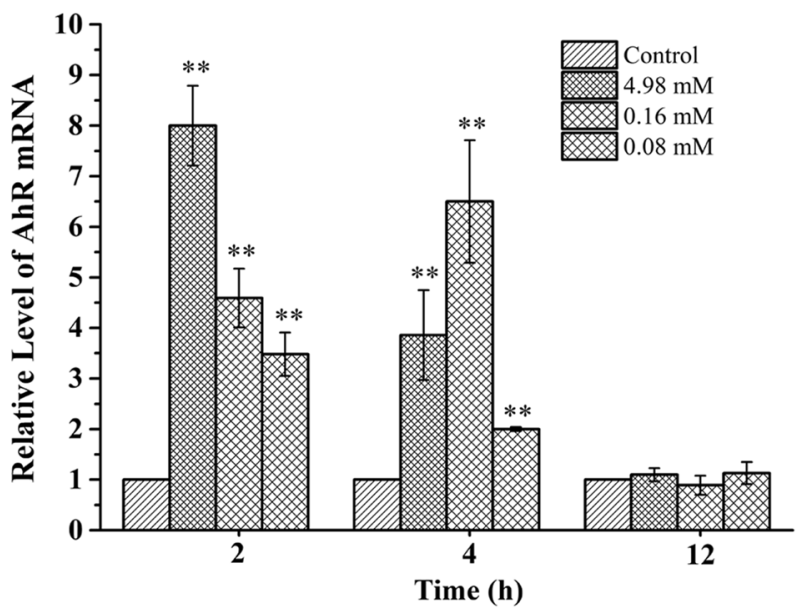

B

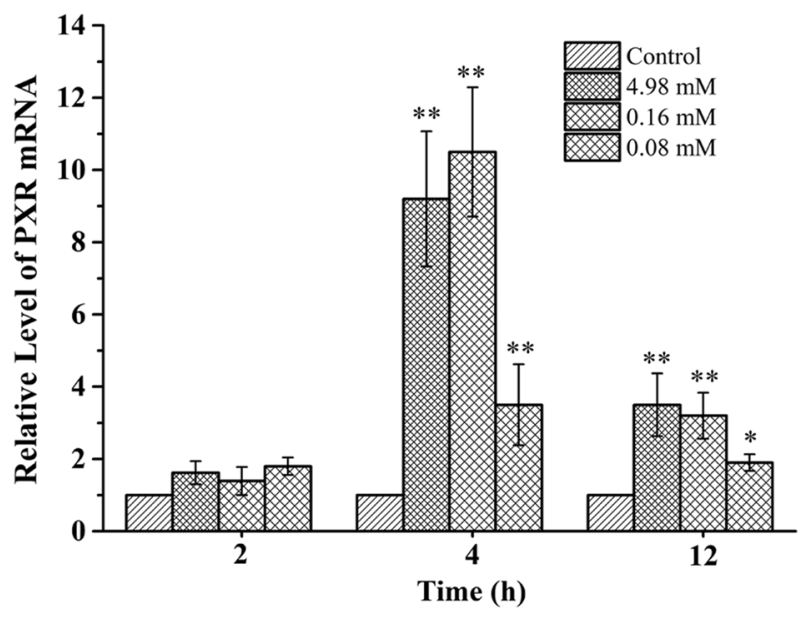

C

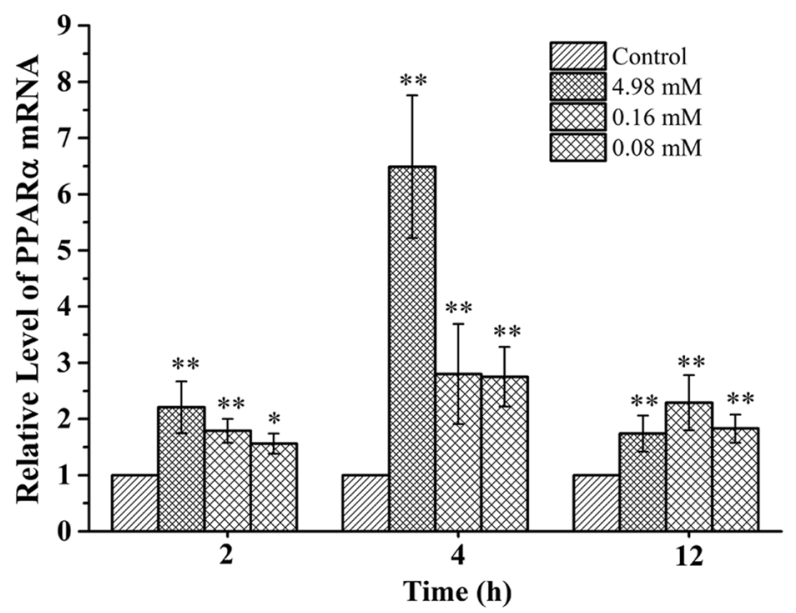

Figure 5. DPTM increase the mRNA expression of AhR (A), PXR (B) and PPARa (C) in Hep G2 cells. Data are the mean $\pm \mathrm{SD}(\mathrm{n}=3) .{ }^{\star} P<0.05$ and ${ }^{\star *} P<0.01$ versus the control group.

\begin{tabular}{|c|c|c|c|c|c|c|c|c|c|c|c|}
\hline \multirow[b]{2}{*}{ Substance } & \multirow[b]{2}{*}{ Dose $(\mu \mathrm{mol} /$ plate $)$} & \multicolumn{2}{|l|}{ TA97 } & \multicolumn{2}{|l|}{ TA98 } & \multicolumn{2}{|l|}{ TA100 } & \multicolumn{2}{|l|}{ TA102 } & \multicolumn{2}{|l|}{ TA1535 } \\
\hline & & $+\mathrm{S} 9$ & - S9 & $+\$ 9$ & $-S 9$ & $+\mathrm{S9}$ & - S9 & $+\$ 9$ & - S9 & $+\mathrm{S9}$ & $-S 9$ \\
\hline \multirow{5}{*}{ DPTM } & 3.98 & $182 \pm 12$ & $169 \pm 14$ & $30 \pm 7$ & $28 \pm 11$ & $142 \pm 22$ & $173 \pm 15$ & $231 \pm 19$ & $268 \pm 12$ & $16 \pm 3$ & $22 \pm 4$ \\
\hline & 1.99 & $184 \pm 13$ & $174 \pm 17$ & $29 \pm 8$ & $32 \pm 8$ & $145 \pm 14$ & $170 \pm 12$ & $245 \pm 20$ & $264 \pm 15$ & $13 \pm 5$ & $22 \pm 3$ \\
\hline & 1.00 & $173 \pm 13$ & $171 \pm 8$ & $33 \pm 9$ & $27 \pm 8$ & $151 \pm 18$ & $165 \pm 14$ & $223 \pm 12$ & $260 \pm 17$ & $14 \pm 3$ & $26 \pm 1$ \\
\hline & 0.50 & $168 \pm 17$ & $165 \pm 14$ & $27 \pm 11$ & $30 \pm 10$ & $140 \pm 10$ & $162 \pm 13$ & $227 \pm 9$ & $241 \pm 10$ & $19 \pm 4$ & $21 \pm 5$ \\
\hline & 0.25 & $175 \pm 17$ & $70 \pm 11$ & $29 \pm 6$ & $33 \pm 6$ & $165 \pm 14$ & $171 \pm 15$ & $235 \pm 20$ & $256 \pm 20$ & $15 \pm 2$ & $22 \pm 3$ \\
\hline \multicolumn{2}{|l|}{\begin{tabular}{|l|} 
Negative control \\
\end{tabular}} & $181 \pm 20$ & $159 \pm 12$ & $28 \pm 6$ & $31 \pm 6$ & $160 \pm 11$ & $169 \pm 11$ & $231 \pm 26$ & $250 \pm 25$ & $19 \pm 2$ & $22 \pm 4$ \\
\hline \multicolumn{2}{|l|}{ Positive control } & $2,986 \pm 209$ & $803 \pm 37$ & $1,464 \pm 348$ & $164 \pm 16$ & $1,071 \pm 133$ & $984 \pm 59$ & $2008 \pm 157$ & $690 \pm 33$ & $347 \pm 21$ & $774 \pm 20$ \\
\hline
\end{tabular}

Table 2. Ames test results of DPTM using S. typhimurium strains TA97, TA98, TA100, TA102 and TA1535. The numbers indicate the means and standards deviation values of CFU in triplicate assay systems. + S9: with metabolic activation; - S9: without metabolic activation. Negative control: DMSO (0.2 mL/plate). Positive control: 2-aminoanthracene for all strains with S9; 4-nitro-o-phenylenediamine for TA97 without S9; daunorubicin for TA98 without S9, sodium azide for TA 100 and TA 1535 without S9, mitomycin for TA102 without S9. 
significantly increased the mRNA expressions of CYP isoforms and their transcriptional regulators when HepG2 cells were treated for a certain period of time. The Ames test showed that DPTM did not exhibit mutagenic response under the present experimental conditions. These results provide useful data and information for further developing DPTM to a potential anti-bacterial clinical candidate.

\section{Material and methods}

Materials. DPTM was synthesized in our laboratory using the standard technique ${ }^{16}$. The purity of this compound was checked by HPLC and quantitative NMR analyses with 98.72\% (see Supplementary S1), and its structure was confirmed by IR, NMR and HR-MS spectrometry. Acetonitrile, methyl alcohol and formic acid were of liquid chromatography-mass spectrometry (LC-MS) grade and purchased from Fisher Scientific (New Jersey, USA). Ultrapure water was purchased from A.S. Watson Group (Guangzhou) Ltd. (Guangzhou, China) and used throughout the study. Tolbutamide (CAS No. 64-77-7), metoprolol tartrate (CAS No. 56392-17-7), phenacetin (CAS No. 62-44-2), chlorzoxazone (CAS No. 95-25-0), dapsone (CAS No. 80-08-0), ketoconazole (CAS No. 65277-42-1), fluvoxamine (CAS No. 54739-18-3), glucose-6-phosphate (CAS No. 5996-17-8), glucose-6-phosphate dehydrogenase (CAS No. 9001-40-5) and nicotinamide adenine dinucleotide phosphate (NADP, CAS No. 53-59-8) were purchased from Sigma-Aldrich (St. Louis, USA). 2-Aminoanthracene (CAS No. 613-13-8) was purchased from Wako Pure Chemical Industries (Chuo-ku, Japan). 4-Nitro-o-phenylenediamine (CAS No. 99-56-9), daunorubicin (CAS No. 23214-92-8), sodium azide (CAS No. 26628-22-8) and mitomycin (CAS No. 99-56-9) were purchased from Acros Organics (Trenton, USA). Trizol reagent was purchased from Beijing Suo Laibao Technology Co., Ltd. (Beijing, China). Diethylpyrocarbonate (DEPC) treated water was purchased from Leagene Biotechnology (Beijing, China). All the other chemicals were of analytical grade and obtained from commercial sources.

Hep G2 cells were purchased from Beina Biotechnology Co., Ltd. (Beijing, China). RPMI-1640 culture media and SYBR Green Real-time PCR Master Mix were purchased from Thermo Fisher Scientific (Carlsbad, USA). Foetal bovine serum was purchased from Bovogen Biologicals Pty Ltd (East Keilor, Australia). Male Pooled SD Rat Liver S9, male SD rat liver microsomes and pooled human liver microsomes were all procured from Research Institute for Liver Diseases (Shanghai) Co., Ltd (Shanghai, China). PrimeScript RT reagent Kit was purchased from Takara Biotech Co., Ltd. (Kusatsu, Japan). Salmonella typhimurium (S. typhimurium) strains TA97, TA98, TA100, TA102 and TA1535 were purchased from Molecular Toxicology, Inc. (MolTox), USA (Blue Ridge Mountains, USA) $)^{35}$.

Methods. Culturing of Hep G2 and cytotoxicity assay. Intrinsic toxicity of DPTM was conducted as described in the literature ${ }^{36-38}$ with some modifications. Briefly, $100 \mu \mathrm{L} / \mathrm{mL}$ of RPMI- 1640 medium containing $10 \%$ foetal bovine serum and Hep G2 cells was seeded in each well of a flat-bottom 96-well plate. After proliferating to logarithmic growth phase at $37^{\circ} \mathrm{C}$ under $5 \% \mathrm{CO}_{2}$, cells were seeded to a new 96-well plate with a density of $4 \times 10^{6}$ cells/hole and cultured for $24 \mathrm{~h}$ at the same condition. The media were replaced with $100 \mu \mathrm{L}$ of serial dilutions of DPTM (10 mg DPTM was dissolved in $1 \mathrm{~mL} 1 \%$ DMSO to a solution and then diluted two-fold with distilled water to provide $19.91,9.96,4.98,2.49,1.25,0.62,0.31,0.16$ and $0.08 \mathrm{mM}$ serial dilutions). The blank group only used $1 \%$ DMSO to replace the media. The obtained mixture was incubated for $24 \mathrm{~h}$. After that, all media in cells were removed and $20 \mu \mathrm{L}$ of MTT reagent was added for an additional $4 \mathrm{~h}$. Then, supernatant fraction was removed and $100 \mu \mathrm{L}$ dimethyl sulfoxide (DMSO) was added, followed by incubation at $37{ }^{\circ} \mathrm{C}$ for $15 \mathrm{~min}$. The absorbance of each well was determined by a spectrophotometer at dual wavelengths of 570 and $279 \mathrm{~nm}$ for the background. The viability percentage was calculated by the following formula: cell viability $\%=\left(O D_{\text {sample }}-\right.$ $\left.\mathrm{OD}_{\text {blank }} / \mathrm{OD}_{\text {control }}-\mathrm{OD}_{\text {blank }}\right) \times 100$. A sigmoid-shaped curve was fitted to the data, and the $\mathrm{IC}_{50}$ was calculated by fitting the Hill equation to the data with nonlinear regression (least-squares best fit modelling) using GraphPad Prism 4 (GraphPad Software Inc., San Diego, USA). The measurements were repeated at least three times.

In vitro assay of CYP activity in RLMs and HLMs. We used the cocktail method to study the inhibition activities of DPTM against CYP enzymes in RLMs and HLMs with slight modification ${ }^{39}$. DPTM $(250 \mu \mathrm{mol})$ was dissolved in $1.25 \mathrm{~mL}$ methanol to a solution and then diluted with methanol and water to 2, 10, 20, 100, 200, 400, 1,000 and $2000 \mu \mathrm{M}$, respectively. The obtained serial dilutions $(10 \mu \mathrm{L})$ were added to the incubation mixtures (200 $\mu \mathrm{L}$ total volume), containing $100 \mu \mathrm{L}$ RLMs or HLMs (final concentration was $5 \mu \mathrm{g} / \mu \mathrm{L}$, respectively), $10 \mu \mathrm{L}$ glucose-6-phosphate (final concentration was $1 \mathrm{mM}$ ), $10 \mu \mathrm{L}$ glucose-6-phosphate dehydrogenase (final concentration was $0.1 \mathrm{U} / \mathrm{L}$ ), $20 \mu \mathrm{L}$ potassium phosphate buffer (final concentration was $0.01 \mathrm{M}$ ) and $20 \mu \mathrm{L} \mathrm{MgCl}_{2}$ (final concentration was $2 \mathrm{mM}$ ), to give the final concentrations were $0.1,0.5,1,510,20,50$, and $100 \mu \mathrm{M}$, respectively. For the groups that in the absence of DPTM, $10 \mu \mathrm{L}$ methanol (1\%) replaced the DPTM and was added to the incubation mixtures. The substrates (phenacetin for CYP1A2, tolbutamide for CYP2C9/11, metoprolol for CYP2D1/6, chlorzoxazone for CYP2E1 and dapsone for CYP3A/4) were dissolved methanol and added at final concentrations of $10 \mu \mathrm{M}$ ( $55 \mu \mathrm{M}$ for dapsone). The final concentration of methanol in all the incubation mixtures, including the blank group, was less than $0.2 \%(\mathrm{v} / \mathrm{v})$. After being pre-incubated at $37^{\circ} \mathrm{C}$ for $5 \mathrm{~min}$, the reaction was initiated by adding $20 \mu \mathrm{L}$ NADPH with the final concentration of $1 \mathrm{mM}$. The reaction was quenched by adding $400 \mu \mathrm{L}$ of acetonitrile after being incubated for $15 \mathrm{~min}$. The mixtures were centrifuged at $10,000 \mathrm{rpm}$ for $10 \mathrm{~min}$, and an aliquot of supernatant was transferred to an auto-injector vial for analyzing the five substrates using a previously validated HPLC method ${ }^{40}$. The determined metabolic substrates were used for calculating the relative enzyme activity $\left(\mathrm{E}_{\text {rel }}\right)$ by the following formula: $\mathrm{E}_{\text {rel }}(\%)=\mathrm{Ci}(\mathrm{n}) / \mathrm{Ci}(0) \times 100 \%$, in which $\mathrm{Ci}(\mathrm{n})$ was the determined metabolic substrates in the presence of varying concentrations of DPTM and $\mathrm{Ci}(0)$ was the determined metabolic substrates in the absence of DPTM. According to the obtained relative enzyme activities and inhibitor concentrations, the $\mathrm{IC}_{50}$ values were obtained as the same method as the cytotoxicity of 


\begin{tabular}{|c|c|}
\hline Gene Name & Sequence $\left(5^{\prime} \rightarrow 3^{\prime}\right)$ \\
\hline \multirow{2}{*}{ GAPDH } & FP: AAGAAGGTGGTGAAGCAGGC \\
\hline & RP: GCGTCAAAGGTGGAGGAGTG \\
\hline \multirow{2}{*}{ CYP2B6 } & FP: CGGATTCAGGAGGAGGCT \\
\hline & RP: GCAGATGATGTTGGCGGT \\
\hline \multirow{2}{*}{ CYP2C8 } & FP: AATGGAAAGAGATGGAAGGAGAT \\
\hline & RP: AGCACAGCCCAGGATGAAAG \\
\hline \multirow{2}{*}{ CYP2C9 } & FP: GGGACAGAGACGACAAGCA \\
\hline & RP: TGTAGCACAGAAGTCAGGGAAA \\
\hline \multirow{2}{*}{ CYP2C19 } & FP: TCCCAAGGGCACAACCATA \\
\hline & RP: CCTCTCCCACACAAATCCG \\
\hline \multirow{2}{*}{ CYP1B1 } & FP: CACCTCTGTCTTGGGCTACC \\
\hline & RP: ACTGAAAAAATCATCACTCTGCT \\
\hline \multirow{2}{*}{ CYP3A4 } & FP: CGAAGATACACAAAAGCACCGA \\
\hline & RP: TTCATAGCCAGCAAAAATAAAGATAA \\
\hline \multirow{2}{*}{ CYP3A5 } & FP: ATTCCTTACCCCAGTTTTTGA \\
\hline & RP: GGTGCTTTTGTTTGTCGTTG \\
\hline \multirow{2}{*}{ CYP2D6 } & FP: GCAAGGTCCTACGCTTCCAA \\
\hline & RP: CTCAGTCAGGTCTCGGGGG \\
\hline \multirow{2}{*}{ CYP1A1 } & FP: GCCTCTGTCATCTTCTGTCTGG \\
\hline & RP: CATACTGCTGGCTCATCCTTG \\
\hline \multirow{2}{*}{ AHR } & FP: CTTTATTGTGCCGAGTCCCA \\
\hline & RP: CTCTGTTCCTTCCTCATCTGTTAGT \\
\hline \multirow{2}{*}{ CAR } & FP: CTCCTGCTGTGCTTCGTGCT \\
\hline & RP: GCAGTTTCCCCTTTGGCTTT \\
\hline \multirow{2}{*}{ PXR } & FP: CGGAAGAAAAGTGAACGGACA \\
\hline & RP: TCGGAGAAGGTAGTGTCAAAGGT \\
\hline \multirow{2}{*}{ PPAR $\alpha$} & FP: GTCATCACGGACACGCTTT \\
\hline & RP: CCCCGCAGATTCTACATTC \\
\hline
\end{tabular}

Table 3. Primer and sequence for real-time PCR. $F P$ forward primer, $P R$ reverse primer, $A h R$ aryl hydrocarbon receptor, $P X R$ pregnane $\mathrm{X}$ receptor, $P P A R \alpha$ peroxisome proliferator-activated receptor $\alpha$.

DPTM. Various concentrations of fluvoxamine (final concentrations were $0.02,0.05,0.1,0.5,1,510$ and $20 \mu \mathrm{M}$ ) and ketoconazole (final concentrations were $0.01,0.02,0.05,0.1,0.5,1,5$ and $10 \mu \mathrm{M}$ ) which replaced DPTM for CYP1A2 and CYP3A4 were performed as the same procedure. The same procedure was repeated three times.

Specific mRNA expression in Hep G2 cells. The logarithmic growth phase Hep G2 cells were seeded into 12-well plastic plates at a density of $4 \times 10^{7}$ cells/well. After incubation at $37^{\circ} \mathrm{C}$ under $5 \% \mathrm{CO}_{2}$ for $24 \mathrm{~h}$, the media were then replaced with $1 \mathrm{~mL}$ of serial dilutions of the DPTM $(4.98,0.16$ and $0.08 \mathrm{mM})$ in fresh media. When cells were incubated for 2,4 and $12 \mathrm{~h}$ at $37^{\circ} \mathrm{C}$ under $5 \% \mathrm{CO}_{2}$, the RNA was isolated using the Trizol reagent according to the manufacturer's protocol. Total RNA $(5 \mu \mathrm{L})$ was transcribed into cDNA by using the PrimeScript RT reagent Kit (Perfect Real Time). The obtained cDNA $(5 \mu \mathrm{L})$ was added for 45 cycles of PCR amplification to detect the mRNA expression (the primers and sequences used herein were given in Table 3) using the ABI Villa TM7Dx real-time PCR system (Gaithersburg, USA). Glyceraldehyde-3-phosphate dehydrogenase (GAPDH) was used as reference gene. All reactions were performed in a final volume of $20 \mu \mathrm{L}(10 \mu \mathrm{L}$ of $2 \times$ SYBR Green Real-time PCR Master Mix, $2 \mu \mathrm{L}$ of cDNA and $2 \mu \mathrm{L}$ of the primers and $6 \mu \mathrm{L}$ DEPC water) and executed in triplicate.

Ames test. S. typhimurium strains TA97, TA98, TA100, TA102 and TA1535 were stored and maintained as provided in the standard protocol ${ }^{41}$. Each of the strains was checked for their genetic integrity prior to the experiment, and tested for biotin dependence, histidine dependence, biotin and histidine dependence, $r f a$ marker (crystal violet) and presence of the plasmid pKM101 (ampicillin resistance) or PAQ1 (tetracycline resistance). Different strains were used to identify different types of mutations.

The test was carried out at five different concentrations of DPTM. DPTM $(39.84 \mu \mathrm{mol})$ was weighed accurately and dissolved in $2 \mathrm{~mL}$ DMSO. Then $1 \mathrm{~mL}$ solution was diluted with distilled water by two fold. The obtained serial dilutions $(1 \mathrm{~mL})$ were then incorporated into $9 \mathrm{~mL}$ melted top agar to dilute to the desired concentrations $(1.99,1.00,0.50,0.25$ and $0.13 \mu \mathrm{mol} / \mathrm{mL})$. The negative control used in this experiment was $1 \mathrm{~mL}$ DMSO which was directly incorporated into $9 \mathrm{~mL}$ melted top agar. 2-aminoanthracene was used as positive control for all bacterial strains in the presence of $S 9$ with the concentration of $13.3 \%(\mathrm{v} / \mathrm{v})$. Four different positive control chemicals were used for each tested strain without S9, including 4-nitro-o-phenylenediamine (10 $\mu \mathrm{g} / \mathrm{plate})$ for 
TA97, daunorubicin ( $2 \mu \mathrm{g} /$ plate) for TA98, sodium azide ( $5 \mu \mathrm{g} /$ plate) for TA 100 and TA 1535, and mitomycin $(0.5 \mu \mathrm{g} /$ plate $)$ for TA102.

The Ames test of DPTM was conducted as described in the literatures ${ }^{42,43}$ with some modifications. Briefly, $1 \mathrm{~mL}$ of strain was inoculated into $5 \mathrm{~mL}$ of nutrient broth, followed by incubating overnight at $37^{\circ} \mathrm{C}$. The mixture comprised respective strains, drug, negative and positive controls with the $S 9$ for testing in the presence of metabolic activation system. For testing without $S 9,1 \times$ phosphate buffer saline $(\mathrm{PBS}, \mathrm{pH} 7.4)$ replaced the $S 9$ mix. The obtained mixtures were added into the tube, mixed well and incubated at $37^{\circ} \mathrm{C}$ for $20 \mathrm{~min}$. Then, the top agar was melted and $2 \mathrm{~mL}$ of this melted top agar was added to each tube and gently mixed. The mixture was poured onto the surface of glucose minimal (GM) agar plate (the final concentrations of DPTM were 3.98, 1.99, $1.00,0.50$ and $0.25 \mu \mathrm{mol} /$ plate and the final concentration of DMSO was $0.2 \mathrm{~mL} /$ plate, respectively). The plate was then swirled to distribute the overlay agar to all surfaces of GM agar, followed by invertion and incubation at $37^{\circ} \mathrm{C}$ for $48 \mathrm{~h}$ when the top agar solidified. The resultant colonies were counted manually considering the high density of bacterial colony. The results were expressed as the number of revertant colonies per plate. The experiments were performed in triplicate.

Data analysis. The data were presented as mean \pm standard deviation (SD). Statistical analyses were performed by One-way ANOVA followed by the Line Segment Detector (LSD) test using SPSS 17.0 software. Differences were considered significant at $p<0.05$.

Received: 23 July 2019; Accepted: 3 June 2020

Published online: 10 August 2020

\section{References}

1. Kavanagh, F., Hervey, A. \& Robbins, W. J. Antibiotic substances from basidiomycetes: VIII. Pleurotus multilus (Fr.) Sacc. and Pleurotus Passeckerianus pilat. Proc. Natl. Acad. Sci. USA 37, 570-574 (1951).

2. Novak, R. \& Shlaes, D. M. The pleuromutilin antibiotics: a new class for human use. Curr. Opin. Invest Drugs 11, 182-191 (2010).

3. Schlunzen, F., Pyetan, E., Fucini, P., Yonath, A. \& Harms, J. M. Inhibition of peptide bond formation by pleuromutilins: the structure of the 50S ribosomal subunit from Deinococcus radiodurans in complex with tiamulin. Mol. Microbiol. 54, 1287-1294 (2004).

4. Davidovich, C. et al. Induced-fit tightens pleuromutilins, binding to ribosomes and remote interactions enable their selectivity. Proc. Natl. Acad. Sci. USA 104, 4291-4296 (2007).

5. Burch, D. G. Tiamulin activity against Brachyspira hyodysenteriae. Vet. Rec. 163, 760 (2008).

6. Stipkovits, L. et al. The efficacy of valnemulin (Econor) in the control of disease caused by experimental infection of calves with Mycoplasma bovis. Res. Vet. Sci. 78, 207-215 (2005).

7. Scangarella-Oman, N. E., Shawar, R. M., Bouchillon, S. \& Hoban, D. Microbiological profile of a new topical antibacterial: retapamulin ointment 1\%. Expert. Rev. Anti Infect. Ther. 7, 269-279 (2009).

8. Nagabushan, H. Retapamulin: A novel topical antibiotic. Indian J. Dermatol. Venereol. Leprol. 76, 77-79 (2010).

9. FDA. FDA approves new antibiotic to treat community-acquired bacterial pneumonia https://www.fda.gov/news-events/press -announcements/fda-approves-new-antibiotic-treat-community-acquired-bacterial-pneumonia (2019).

10. Waites, K. B. et al. In vitro activities of lefamulin and other antimicrobial agents against macrolide-susceptible and macrolideresistant Mycoplasma pneumoniae from the United States, Europe, and China. Antimicrob. Agents Chemother. 61, e02008-e2016 (2017).

11. Shang, R. F. et al. Synthesis and biological activities of novel pleuromutilin derivatives with a substituted thiadiazole moiety as potent drug-resistant bacteria inhibitors. J. Med. Chem. 57, 5664-5678 (2014).

12. Stresser, D. M. et al. Highly selective inhibition of human CYP3A in vitro by azamulin and evidence that inhibition is irreversible. Drug Metab. Dispos. 32, 105-112 (2004).

13. Gao, M. L. et al. Design, synthesis and antibacterial evaluation of novel pleuromutilin derivatives possessing piperazine linker. Eur. J. Med. Chem. 127, 286-295 (2017).

14. Witkamp, R. F., Nijmeijer, S. M. \& Van Miert, A. S. Cytochrome P-450 complex formation in rat liver by the antibiotic tiamulin. Antimicrob. Agents Chemother. 40, 50-54 (1996).

15. Ling, C. Y. et al. Design, synthesis, and structure-activity relationship studies of novel thioether pleuromutilin derivatives as potent antibacterial agents. J. Med. Chem. 57, 4772-4795 (2014).

16. Yi, Y. P. et al. Synthesis and antibacterial activities of novel pleuromutilin derivatives with a substituted pyrimidine moiety. Eur. J. Med. Chem. 126, 687-695 (2017).

17. Goethe, O. et al. Antibacterial properties and clinical potential of pleuromutilins. Nat. Prod. Rep. https://doi.org/10.1039/C8NP0 0042E (2018).

18. Novak, R. Are pleuromutilin antibiotics finally fit for human use?. Ann. NY Acad. Sci. 1241, 71-81 (2011).

19. Lee, K. S. \& Kim, S. K. Direct and metabolism-dependent cytochrome P450 inhibition assays for evaluating drug-drug interactions. J. Appl. Toxicol. 33, 100-108 (2013).

20. Noushad, M., Kannan, T. P., Husein, A., Abdullah, H. \& Ismail, A. R. Genotoxicity evaluation of locally produced dental porcelain -an in vitro study using the Ames and Comet assays. Toxicol. In Vitro 23, 1145-1150 (2009).

21. Nebert, D. W. \& Russell, W. Clinical importance of the cytochromes P450. Lancet 360, 1155-1162 (2002).

22. Denisov, I. G., Makris, T. M., Sligar, S. G. \& Schlichting, I. Structure and chemistry of cytochrome P450. Chem. Rev. 36, 2253-2277 (2005).

23. Basheer, L., Schultz, K. \& Kerem, Z. Inhibition of cytochrome P450 3A by acetoxylated analogues of resveratrol in in vitro and in silico models. Sci. Rep. 17, 31557-31569 (2016).

24. Pergolizzi, J. V. et al. Prevalence of exposure to potential CYP450 pharmacokinetic drug-drug interactions among patients with chronic low back pain taking opioids. Pain. Pract. 11, 230-239 (2011).

25. Chen, N. et al. The oral bioavailability, excretion and cytochrome $\mathrm{P} 450$ inhibition properties of epiberberine: an in vivo and in vitro evaluation. Drug Des. Devel. Ther. 28, 57-65 (2018).

26. Jacobs, M. R. Retapamulin: a semisynthetic pleuromutilin compound for topical treatment of skin infections in adults and children. Future. Microbiol. 2, 591-600 (2007).

27. Ai, X. et al. Synthesis and pharmacological evaluation of novel pleuromutilin derivatives with substituted benzimidazole moieties. Molecules 21, 1488 (2016). 
28. Witkamp, R. F., Nijmeijer, S. M. \& Van-Miert, A. S. Cytochrome P-450 complex formation in rat liver by the antibiotic tiamulin. Antimicrob. Agents Chemother. 40, 50-54 (1996).

29. Ling, C. Y. et al. Design, synthesis, and structure activity relationship studies of novel thioether pleuromutilin derivatives as potent antibacterial agents. J. Med. Chem. 57, 4772-4795 (2014).

30. Rasmussen, M. K., Bertholdt, L., Gudiksen, A., Pilegaard, H. \& Knudsen, J. G. Impact of fasting followed by short-term exposure to interleukin-6 on cytochrome p450 mRNA in mice. Toxicol. Lett. 282, $93-99$ (2018).

31. Pavek, P. \& Dvorak, Z. Xenobiotic-induced transcriptional regulation of xenobiotic metabolizing enzymes of the cytochrome P450 superfamily in human extrahepatic tissues. Curr. Drug Metab. 9, 129-143 (2008).

32. Fu, Y. X. et al. A validated HPLC-MS/MS assay for 14-O-[(4,6-Diaminopyrimidine-2-yl)thioacetyl] mutilin in biological samples and its pharmacokinetic, distribution and excretion via urine and feces in rats. Molecules 24, 790 (2019).

33. Pukalskiené, M. et al. Genotoxicity and antioxidant activity of five agrimonia and filipendula species plant extracts evaluated by comet and micronucleus assays in human lymphocytes and ames salmonella/microsome test. Food Chem. Toxicol. 113, 303-313 (2018).

34. Li, J., Kong, X., Li, X., Yang, Y. \& Zhang, J. Genotoxic evaluation of aspirin eugenol ester using the Ames test and the mouse bone marrow micronucleus assay. Food Chem. Toxicol. 62, 805-809 (2013).

35. Mortelmans, K. \& Zeiger, E. The AMES salmonella/microsome mutagenicity assay. Mutat. Res. 455, 29-60 (2000).

36. Koehler, S. \& Wiese, M. HM30181 derivatives as novel potent and selective inhibitors of the breast cancer resistance protein (BCRP/ ABCG2). J. Med. Chem. 58, 3910-3921 (2015).

37. Al-Tamimi, M. A., Rastall, B. \& Abu-Reidah, I. M. Chemical composition, cytotoxic, apoptotic and antioxidant activities of main commercial essential oils in palestine: A comparative study. Medicines 3, e3040027 (2016).

38. Schmitt, S. M., Stefan, K. \& Wiese, M. Pyrrolopyrimidine derivatives as novel inhibitors of multidrug resistance-associated protein 1 (MRP1, ABCC1). J. Med. Chem. 59, 3018-3033 (2016).

39. Li, G., Huang, K. \& Breemen, R. B. High-throughput cytochrome P450 cocktail inhibition assay for assessing drug-drug and drug-botanical interactions. Drug. Metab. Dispos. 43, 1670-1678 (2015).

40. Wei, L. Y. et al. Effect of comparability of Coptis chinensis and Scutellaria baicalensis on five sub enzymatic activities of liver microsomes in rats. Chin. J. Chin. Mater. Med. 38, 1426-1429 (2013).

41. OECD. Organisation for economic co-operation and development, guideline for testing of chemicals: Bacterial reverse mutation test, p. 471 (1997).

42. Wahab, N. F. A. C., Kannan, T. P., Mahmood, Z., Rahman, I. A. \& Ismail, H. Genotoxicity assessment of biphasic calcium phosphate of modified porosity on human dental pulp cells using Ames and comet assays. Toxicol. In Vitro 47, 207-212 (2018).

43. Nelma, D. M. et al. In vitro mutagenicity assay (Ames test) and phytochemical characterization of seeds oil of helianthus annuus linné (sunflower). Toxicol. Rep. 3, 733-739 (2016).

\title{
Acknowledgements
}

This work was supported by the National Natural Science Foundation of China (No. 31873027), National Key Technology Support Program (No. 2015BAD11B02) and Agricultural Science and Technology Innovation Program (ASTIP, No. CAASASTIP-2014-LIHPS-04).

\section{Author contributions}

Y.F. and R.S. designed the experimental; Y.F., Y.Y. and Y.F. performed the experiments; Y.F., Y.Y. and R.S. analysed and interpreted the data; R.S. wrote the manuscript. All authors read the manuscript.

\section{Competing interests}

The authors declare no competing interests.

\section{Additional information \\ Supplementary information is available for this paper at https://doi.org/10.1038/s41598-020-70400-8.}

Correspondence and requests for materials should be addressed to R.S.

Reprints and permissions information is available at www.nature.com/reprints.

Publisher's note Springer Nature remains neutral with regard to jurisdictional claims in published maps and institutional affiliations.

\begin{abstract}
Open Access This article is licensed under a Creative Commons Attribution 4.0 International License, which permits use, sharing, adaptation, distribution and reproduction in any medium or format, as long as you give appropriate credit to the original author(s) and the source, provide a link to the Creative Commons license, and indicate if changes were made. The images or other third party material in this article are included in the article's Creative Commons license, unless indicated otherwise in a credit line to the material. If material is not included in the article's Creative Commons license and your intended use is not permitted by statutory regulation or exceeds the permitted use, you will need to obtain permission directly from the copyright holder. To view a copy of this license, visit http://creativecommons.org/licenses/by/4.0/.
\end{abstract}

(c) The Author(s) 2020 